\title{
Barriers to Adolescent Immunization: A Survey of Family Physicians and Pediatricians
}

\author{
Natalia Vukshich Oster, RN, MPH; Carol A. McPhillips-Tangum, MPH, * \\ Francisco Averhoff, MD, MPH, and Kelly Howell, MPH
}

Background: Although early childhood vaccination rates have increased, many adolescents are not up to date on recommended vaccinations. We assessed attitudes and practices of family physicians and pediatricians regarding adolescent vaccination to identify provider-level barriers that may contribute to low immunization rates.

Methods: A 94-item self-report questionnaire was mailed to 400 physicians contracted with a managed care organization. Physicians were queried about demographic characteristics, source of vaccine recommendations, adolescent immunization practices, barriers to immunizing adolescents, and use of reminder/recall systems.

Results: Response rate was 59\%. Most respondents reported routinely recommending vaccines for tetanus and diphtheria toxoids $(98 \%)$, Hepatitis B $(90 \%)$, and measles, mumps, and rubella $(84 \%)$, whereas $60 \%$ routinely recommended varicella vaccine. Physicians reported that they were more likely to assess immunization status, administer indicated immunizations, and schedule return immunization visits to younger adolescents (11 to 13 years old) than to older adolescents (14 to 18 and 19 to 21 years old).

Conclusion: Most respondents reported recommending the appropriate vaccinations during preventive health visits; however, older adolescents were least likely to be targeted for immunization assessment and administration of all recommended vaccines. (J Am Board Fam Pract 2005;18:13-9.)

Adolescent immunization rates remain low despite the success of infant and childhood vaccination programs. Although routine adolescent immunizations have been recommended since 1996, an estimated 35 million adolescents (ie, persons 11 to 21 years of age, as defined by the American Medical Association and the American Academy of Pediatrics) lack one or more recommended vaccinations, placing them at risk for developing vaccine-preventable disease. ${ }^{1-3}$ Factors contributing to low immunization rates include missed opportunities, lack

Submitted, revised, 17 August 2004.

From the Emory Center on Health Outcomes and Quality (ECHOQ), Atlanta, Rollins School of Public Health, Atlanta, GA (NVO, CAM-T, KH), and National Immunization Program, Centers for Disease Control and Prevention, Atlanta, GA (FA). Address correspondence to Natalia Vukshich Oster, RN, MPH, Emory Center on Health Outcomes and Quality, Department of Health Policy and Management, 6th Floor, Rollins School of Public Health, 1518 Clifton Road, Atlanta, GA 30322 (e-mail: noster@sph. emory.edu).

* Current affiliation: CMT Consulting, Decatur, GA.

This study was funded in part by Aetna. The opinions expressed and conclusions reached are solely those of the authors and do not necessarily represent those of Aetna. This study was also supported, in part, by a grant from Merck Vaccine Division. of population-based immunization registries that include adolescents, low public and peer awareness about immunization coverage in this age group, misperceptions about vaccine safety, and lack of knowledge about the importance of immunizations. ${ }^{1-4}$ In addition, adolescents are one of the most difficult groups to reach for vaccination and other preventive services, in part because growing numbers lack a primary care provider and adolescents, in general, have fewer contacts with physicians. ${ }^{1,5-6}$

Overcoming barriers at the provider level is crucial to increasing adolescent immunization rates. Previous studies have shown that lack of a provider recommendation is often cited as a reason for not receiving an immunization and that provider attitude and recommendation are strong predictors of vaccination of high-risk patients and indeed may be the most important determinants of immunization status. $^{7-9}$

The Centers for Disease Control and Prevention (CDC) recommend that all previously unvaccinated adolescents receive tetanus and diphtheria (Td) toxoids, varicella vaccine (if no history of natural disease), hepatitis $\mathrm{B}$, and measles, mumps, and 
rubella (MMR) vaccine. In addition, influenza, hepatitis A, and pneumococcal vaccine are recommended for immunocompromised adolescents or those who are at high risk. ${ }^{3}$

This study was designed to assess physician attitudes and practices regarding adolescent vaccination within a managed care organization (MCO) setting and to describe and identify barriers at the provider level that may contribute to low immunization rates.

\section{Methods \\ Study Population}

In May 1999, we obtained a computerized list of all general physicians, family physicians, and pediatricians actively contracted with a national MCO; the list included name, address, phone number, gender, specialty, date of birth, and year of medical school graduation. At the time the survey was conducted, the MCO had 4.9 million members in 28 states and the District of Columbia and contracted with approximately 34,000 physicians. Eligibility criteria included a stated specialty in pediatrics, family practice, or general practice and record of a complete mailing address, including street, city, state, and zip code. Based on a minimum expected response rate of $40 \%$, a sample of 400 physicians was randomly selected to receive a questionnaire. This sample size was chosen to provide a power of $80 \%$ to detect a difference of $20 \%$ between specialties with an $\alpha$ of 0.05 . Questionnaires were sent to physicians in 28 states and the District of Columbia. Physicians did not receive an incentive for participating in the survey.

\section{Questionnaire}

A 94-item self-administered questionnaire was developed, piloted, revised and mailed to each physician in May 1999 by a health care research center affiliated with the MCO. Nonresponders were sent follow-up mailings in June 1999. Respondents returned the questionnaire to the research center via a self-addressed envelope mailed with the survey.

The questionnaire included demographic questions, source of physician's vaccine recommendations, questions regarding adolescent immunization practices, barriers to immunizing adolescents, the degree of importance placed on ensuring that adolescents were up to date (UTD) on immunizations, and tracking or reminder/recall systems used by the provider office. Physicians who did not provide care to adolescent patients were asked to indicate this on the survey and complete only the demographic portion of the survey. Most questions were fixed response; several open-ended questions were included and later converted to multioutcome responses.

Each questionnaire included the name of the physician and a study identification number. Individual identifying information was omitted after receipt of the questionnaire; thus, questionnaires remained confidential, although not anonymous, at all times.

The study design for this project was approved by the Institutional Review Board of The Prudential Center for Health Care Research. (When this research was conducted, the Emory Center on Health Outcomes and Quality was known as The Prudential Center for Health Care Research, which became the USQA Center for Health Care Research; in 2001, it was transferred to Emory University to form the Emory Center on Health Outcomes and Quality.)

\section{Analysis}

Continuous data were collapsed into categories and categorical data were dichotomized before analysis. Simple associations were tested using a $\chi^{2}$ test of association and corresponding $p$ values are reported. A $p$ value of .05 was used as the level of statistical significance. All analysis was conducted in SAS (version 8.02; SAS Institute Inc, Cary, NC).

\section{Results \\ Attributes of Respondents}

Forty of the 400 physicians who were mailed a questionnaire were ineligible: one physician no longer worked at the practice, 12 indicated that they did not provide care to adolescents, and 27 questionnaires were undeliverable. Two surveys were received from general physicians but were removed from the analysis because of the small number of responses from that specialty. Of the remaining 358 physicians, 210 questionnaires were returned for a response rate of $59 \%$.

Respondents and nonrespondents did not differ significantly with respect to age, gender, or year of medical school graduation but did differ in specialty. Pediatricians [97 of 145 (67\%)] were more likely to return the questionnaire than were family 
Table 1. Demographic Characteristics of Survey Respondents and Nonrespondents*

\begin{tabular}{lcc}
\hline Attribute & $\begin{array}{c}\text { Respondents } \\
{[\text { No. }(\%)]}\end{array}$ & $\begin{array}{c}\text { Nonrespondents } \\
{[\text { No. (\%) }]}\end{array}$ \\
\hline $\begin{array}{l}\text { Specialty } \\
\text { Family practice }\end{array}$ & $113(54)$ & $100(68)$ \\
$\quad$ Pediatrics & $97(46)$ & $48(32)$ \\
Year of medical school & & \\
graduation & & \\
1940 to 1959 & $8(5)$ & $4(4)$ \\
1960 to 1979 & $71(46)$ & $64(39)$ \\
After 1980 & $76(49)$ & $102(72)$ \\
Gender & & $39(28)$ \\
Male & $123(64)$ & \\
Female & $69(36)$ & \\
\hline
\end{tabular}

* Nonrespondent data based on administrative data; respondent based on administrative data plus survey response ( $99 \%$ correspondence).

${ }^{\dagger} P<.05$ for differences between respondents and non-respondents.

physicians [113 of $206(55 \%)](P<.05)$. Demographic characteristics of survey respondents and nonrespondents are shown in Table 1.

\section{Source of Vaccine Recommendations}

Respondents were asked to select all their sources of vaccine recommendations from a checklist. Pediatricians were more likely than family physicians to indicate the American Academy of Pediatrics (99\% vs $48 \%, P<.05$ ) and the Advisory Committee on Immunization Practices (55\% vs $23 \%, P<$ $.05)$ as their source of vaccine recommendations, whereas more family physicians than pediatricians indicated the American Academy of Family Physicians as their source of recommendations ( $81 \%$ vs $7 \%, P<.05$ ) (Table 2).

\section{Vaccines Routinely Recommended for Adolescents}

Of the 210 respondents, $98 \%$ reported that they routinely recommended $\mathrm{Td}$ toxoids, $90 \%$ routinely recommended hepatitis B vaccine, $84 \%$ routinely recommended MMR vaccine, and $60 \%$ routinely recommended varicella vaccine (Table 2). Pediatricians were more likely than family physicians to report recommending hepatitis B (99\% vs $82 \%$, $P<.05)$ and varicella $(80 \%$ vs $42 \%, P<.05)$ vaccines; there were no differences by specialty in recommendation rates for $\mathrm{Td}$ and MMR.

One hundred and thirteen (54\%) respondents indicated that they routinely recommended all 4 vaccines of interest (ie, Td, MMR, hepatitis $B$, and varicella), whereas $64(30 \%)$ routinely recom- mended 3 vaccines, $23(11 \%)$ routinely recommended 2 vaccines, 9 (4\%) routinely recommended only one of the vaccines, and $1(<0.05 \%)$ respondent did not routinely recommend any of the vaccines. More pediatricians than family physicians (72\% vs 38\%) reported that they routinely recommended all 4 vaccines.

\section{Immunization Activities Included in Preventive Health Visits}

When asked what immunization activities (ie, assess immunization status, administer needed immunizations, and schedule return immunization visit) were typically included in preventive health visits for adolescents aged 11 to 13,14 to 18 , and 19 to 21 years, results varied by age group (Table 2). Respondents reported that they conducted more immunization assessments during preventive health visits for adolescents aged 11 to 13 (94\%) and 14 to 18 years $(91 \%)$ compared with adolescents aged 19 to 21 years $(74 \%)$. Likewise, more respondents reported administering needed immunizations to adolescents aged 11 to 13 years (96\%) and 14 to 18 years $(95 \%)$ than to adolescents 19 to 21 years of age $(77 \%)$. However, more family physicians than pediatricians reported that they administered needed immunizations for adolescents aged 19 to 21 years $(84 \%$ vs $69 \%, P<.05)$. Only $60 \%$ of respondents indicated that they scheduled return immunization visits for adolescents aged 19 to 21 years, compared with adolescents aged 11 to 13 years $(78 \%)$ and 14 to 18 years $(74 \%)$.

\section{Proportion of Adolescents UTP on Vaccine- Preventable Disease}

Pediatricians were more likely than family physicians to report that $\geq 75 \%$ of their adolescent patients were up to date on Td toxoids, hepatitis B vaccine, and varicella vaccine, whereas there were no differences between the specialties with respect to receipt of MMR (Table 2). In addition, 14\% of respondents reported that $75 \%$ or more of their adolescent patients were UTD on all 4 vaccinepreventable diseases of interest, $21 \%$ indicated the same for 3 diseases, $30 \%$ for 2 diseases, and 13\% for 1 disease; $21 \%$ reported that $\geq 75 \%$ of their adolescent patients were not UTD on any of the diseases of interest. 


\begin{tabular}{|c|c|c|c|}
\hline & $\begin{array}{c}\text { PED* } \\
{[\mathrm{n}=97(\%)]}\end{array}$ & $\begin{array}{c}\mathrm{FP}^{*} \\
{[\mathrm{n}=113(\%)]}\end{array}$ & $\begin{array}{c}\text { Total } \\
{[\mathrm{n}=210(\%)]}\end{array}$ \\
\hline \multicolumn{4}{|l|}{ Source of vaccine recommendations } \\
\hline American Academy of Pediatrics & $96(99)$ & $54(48)$ & $150(71)$ \\
\hline American Academy of Family Practitioners ${ }^{\dagger}$ & $7(7)$ & $92(81)$ & $99(47)$ \\
\hline Advisory Committee on Immunization Practices ${ }^{\dagger}$ & $53(55)$ & $26(23)$ & $79(38)$ \\
\hline \multicolumn{4}{|l|}{ Vaccines routinely recommended for adolescents } \\
\hline Tetanus/diphtheria & $97(100)$ & $108(96)$ & $205(98)$ \\
\hline Measles, mumps, rubella & $86(87)$ & $90(80)$ & $176(84)$ \\
\hline Hepatitis $\mathrm{B}^{\dagger} \mathrm{P}$ - & $96(99)$ & $93(82)$ & $189(90)$ \\
\hline Varicella $^{\dagger}$ & $78(80)$ & $48(42)$ & $126(60)$ \\
\hline \multicolumn{4}{|c|}{ Immunization activities conducted at preventive health visits } \\
\hline \multicolumn{4}{|c|}{ Immunization activity by age } \\
\hline \multicolumn{4}{|l|}{ Assess immunization status } \\
\hline 11 to 13 years & $95(98)$ & $103(91)$ & $198(94)$ \\
\hline 14 to 18 years & $93(96)$ & $98(87)$ & $191(91)$ \\
\hline 19 to 21 years & $72(74)$ & $84(74)$ & $156(74)$ \\
\hline \multicolumn{4}{|l|}{ Administer needed immunizations } \\
\hline 11 to 13 years & $96(99)$ & $105(93)$ & $201(96)$ \\
\hline 14 to 18 years & $94(97)$ & $105(93)$ & $200(95)$ \\
\hline 19 to 21 years* & $67(69)$ & $95(84)$ & $162(77)$ \\
\hline \multicolumn{4}{|l|}{ Schedule return immunization visits } \\
\hline 11 to 13 years & $82(85)$ & $81(72)$ & $163(78)$ \\
\hline 14 to 18 years & $79(81)$ & $77(68)$ & $156(74)$ \\
\hline 19 to 21 years & $54(56)$ & $72(64)$ & $126(60)$ \\
\hline \multicolumn{4}{|c|}{ Self-report that $\geq 75 \%$ adolescent patients up-to-date on vaccinations } \\
\hline Tetanus/diphtheria $^{\dagger}$ & $73(75)$ & $68(62)$ & $141(69)$ \\
\hline Measles, mumps, rubella & $72(79)$ & $73(65)$ & $145(69)$ \\
\hline Hepatitis $\mathrm{B}^{\dagger}$ & $33(34)$ & $21(19)$ & $54(26)$ \\
\hline Varicella $^{\dagger}$ & $41(42)$ & $21(19)$ & $62(31)$ \\
\hline Use of recall/reminder systems & $27(28)$ & $19(17)$ & $47(22)$ \\
\hline
\end{tabular}

* PED, pediatrician; FP, family physician.

${ }^{\dagger} P<.05$ for differences between family physicians and pediatricians.

\section{Use of Reminder and Recall System}

Only $18 \%$ of family physicians and $28 \%$ of pediatricians reported that their practice used a tracking or reminder/recall system to identify and contact adolescents who were due or overdue for immunizations (Table 2). There were no differences in vaccine recommendations between respondents who used a reminder/recall system and those who did not, except with respect to hepatitis B recommendation. Of the 47 respondents who reported that they used a reminder/recall system, $100 \%$ routinely recommended hepatitis B vaccine, compared with $140(88 \%)$ of 160 who reported that they did not use a recall/reminder system $(P<.05)$.

\section{Importance Placed on Ensuring That Adolescents Are UTP on Immunizations}

Between $77 \%$ and $98 \%$ of respondents (depending on physician specialty and vaccine) indicated that it was "very important" to ensure that adolescents were UTD on Td, MMR, and Hepatitis B, whereas a smaller percentage ( $53 \%$ to $85 \%$ ) indicated the same for varicella vaccine (Table 3). Pediatricians were more likely than family physicians to rate being UTD on hepatitis B (95\% vs $77 \%, P<.05)$ and varicella $(85 \%$ vs $53 \%, P<.05)$ as "very important."

\section{Barriers to Vaccinating Adolescents}

Both pediatricians and family physicians selected "adolescents rarely make preventive health visits" ( $45 \%$ to $74 \%$ depending on physician specialty and adolescent age), "adolescents not aware of need for immunizations" ( $45 \%$ to $67 \%$ ), and "adolescents and/or parents underestimate the risk of vaccinepreventable disease" ( $43 \%$ to $57 \%$ ) as the primary barriers to adolescent immunization (Table 4). Very few respondents (3\% to 9\%) found "obtaining consent per state law" to be a barrier. In addition, only $9 \%$ to $12 \%$ of respondents indicated that adolescent and/or parental refusal of vaccinations was a barrier to immunization.

For all adolescent age groups, more family physicians than pediatricians (59\% to $63 \%$ vs $32 \%$ to 
Table 3. Degree of Importance Placed on Ensuring Adolescents Are Up to Date, by Specialty*

\begin{tabular}{|c|c|c|c|}
\hline & $\begin{array}{l}\text { Not Very } \\
\text { Important } \\
{[\text { No. }(\%)]}\end{array}$ & $\begin{array}{l}\text { Important } \\
{[\text { No. }(\%)]}\end{array}$ & $\begin{array}{c}\text { Very } \\
\text { Important } \\
{[\text { No. }(\%)]}\end{array}$ \\
\hline \multicolumn{4}{|c|}{ Tetanus/diphtheria } \\
\hline $\mathrm{PEDS}^{\dagger}$ & $2(2)$ & $0(0)$ & $94(98)$ \\
\hline $\mathrm{FP}^{\dagger}$ & $3(3)$ & $5(5)$ & $102(93)$ \\
\hline Overall & $5(2)$ & $5(2)$ & $196(95)$ \\
\hline \multicolumn{4}{|c|}{ Measles, mumps, rubella } \\
\hline PEDS & $1(1)$ & $1(1)$ & $95(98)$ \\
\hline $\mathrm{FP}$ & $2(2)$ & $3(3)$ & $105(95)$ \\
\hline Overall & $3(1)$ & $4(2)$ & $200(97)$ \\
\hline \multicolumn{4}{|c|}{ Hepatitis $\mathrm{B}^{\ddagger}$} \\
\hline PEDS & $1(1)$ & $4(4)$ & $92(95)$ \\
\hline $\mathrm{FP}$ & $7(6)$ & $18(16)$ & $85(77)$ \\
\hline Overall & $8(4)$ & $22(11)$ & $177(86)$ \\
\hline \multicolumn{4}{|l|}{ Varicella $^{\ddagger}$} \\
\hline PEDS & $5(5)$ & $9(9)$ & $81(85)$ \\
\hline FP & $19(17)$ & $32(29)$ & $59(54)$ \\
\hline Overall & $24(12)$ & $41(20)$ & $140(68)$ \\
\hline
\end{tabular}

* Overall, $\mathrm{N}=210$; pediatricians, $\mathrm{n}=97$; family physicians, $\mathrm{n}=$ 113.

${ }^{+} \mathrm{PED}$, pediatrician; FP, family physician.

${ }^{\ddagger} P<.05$ for differences between family physicians and pediatricians.

$43 \%, P<.05)$ reported "difficult to obtain verification of previous immunization" as a barrier. For adolescents aged 11 to 13 years, more family physicians indicated "adolescents not aware of need for immunizations" ( $62 \%$ vs $45 \%, P<.05$ ) as a barrier than did pediatricians. In addition, more family physicians reported "adolescents rarely make preventive health visits" ( $74 \%$ vs $63 \%, P<.05)$ and "difficult to determine who needs vaccine" (21\% vs $11 \%, P<.05)$ as barriers for adolescents aged 19 to 21 ; however, more pediatricians than family physicians found "adolescents seek care from other facilities" to be a barrier for adolescents aged 19 to 21 ( $41 \%$ vs $26 \%, P<.05$ ).

With the exception of the barriers "difficult to determine adolescents at risk," "adolescents/parents refuse vaccination," and "obtaining consent per state law," more respondents reported that they experienced barriers for adolescents aged 19 to 21 years compared with adolescents aged 11 to 13 and 14 to 18 years $(P<.05)$. When we stratified by the number of barriers experienced, more family physicians reported experiencing 7 or more barriers for adolescents 19 to 21 than did pediatricians (54\% vs $46 \%, P<.05)$. There were no differences by specialty in the number of barriers for adolescents aged 11 to 13 or 14 to 18 years.

\section{Discussion}

The survey results suggest that most physicians recommend the appropriate vaccinations to their adolescent patients and typically include immunization activities in preventive health visits. Both pediatricians and family physicians report that they experience barriers to adolescent immunization, particularly as adolescents aged. Family physicians reported experiencing more barriers for older adolescents compared with pediatricians; however, it is likely that more adolescents aged 19 to 21 seek care from primary care physicians than from pediatricians.

Respondents, in particular family physicians, consistently placed less importance on varicella vaccine compared with hepatitis $\mathrm{B}, \mathrm{MMR}$, and $\mathrm{Td}$. For example, only $68 \%$ of respondents overall reported that it was "very important" to ensure that adolescents were UTD on protection against varicella, whereas $86 \%$ to $97 \%$ reported the same regarding hepatitis $\mathrm{B}, \mathrm{MMR}$, and $\mathrm{Td}$. In addition, most respondents (84\% to $98 \%$ ) reported that they routinely recommended hepatitis B, MMR, and $\mathrm{Td}$, whereas fewer $(60 \%)$ reported routinely recommending varicella vaccine, even though only $31 \%$ of respondents felt that most (ie, $\geq 75 \%$ ) of their adolescent patients were UTD on their protection against varicella compared with $69 \%$ who reported the same for MMR and Td. This disparity may stem from the fact that varicella vaccine is relatively new-less than 3 years had elapsed between our survey and the CDC's recommendation to administer varicella vaccine to adolescents without a history of natural disease, and the vaccine had only been licensed and available for use in the United States for an additional 15 months. ${ }^{10,11}$ Adherence to varicella vaccine recommendations faces several additional challenges: there is a lack of direct medical cost savings, previous surveys have found that some physicians are concerned about waning immunity after vaccination and may prefer natural disease over vaccination, and varicella is generally a benign illness, with rare complications. In addition, previous studies indicate that physicians are concerned that varicella may shift from being a childhood disease to primarily an adult disease, where the complications are greater. ${ }^{10,12-16}$

To the best of our knowledge, only one previous survey has been conducted assessing physician practices with regard to adolescent immuniza- 


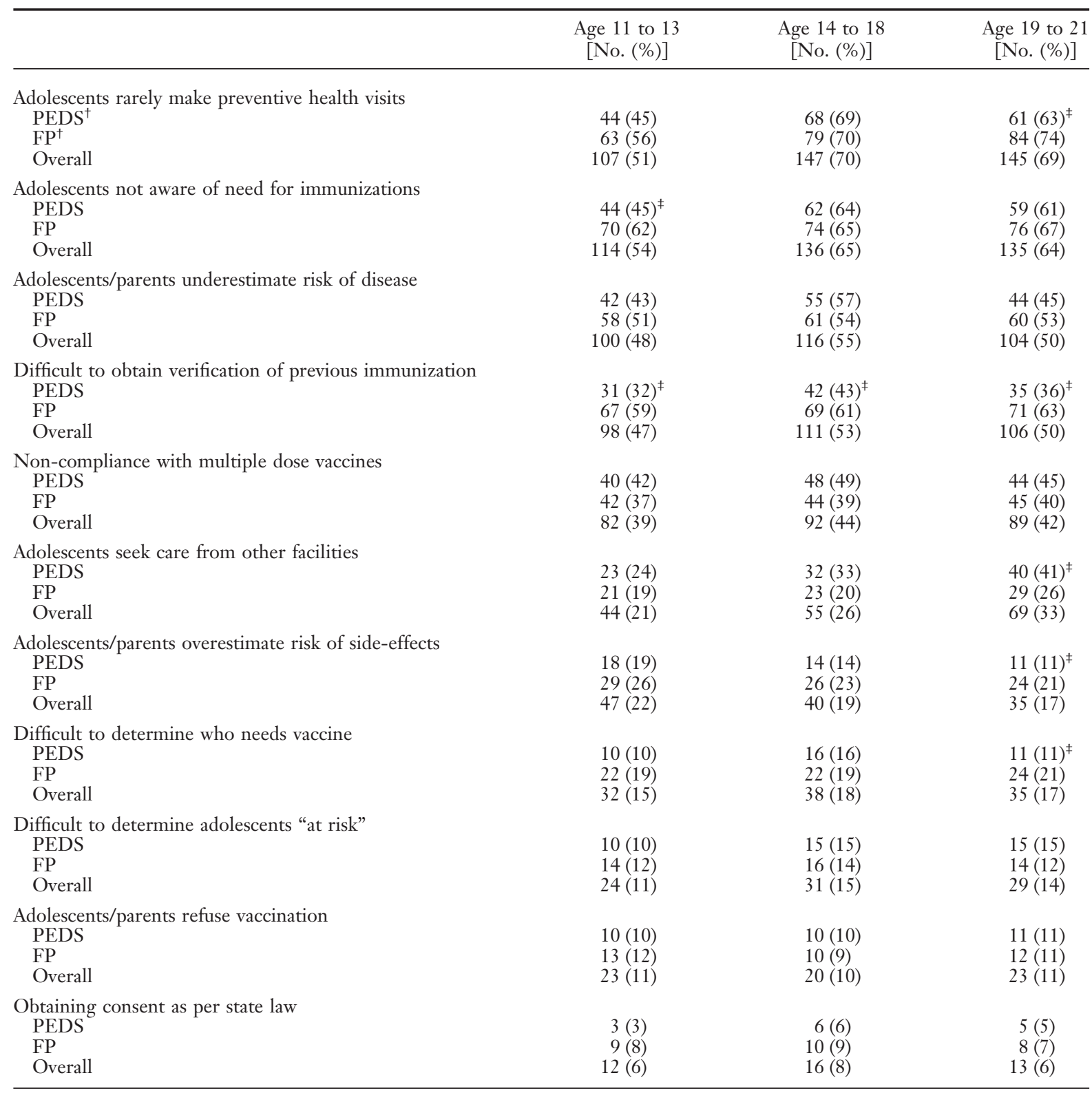

* Overall, $\mathrm{N}=210$; pediatricians, $\mathrm{n}=97$; family physicians, $\mathrm{n}=113$.

${ }^{\dagger} \mathrm{PED}$, pediatrician; FP, family physician.

${ }^{\ddagger} P<.05$ for differences between family physicians and pediatricians.

tion. ${ }^{17}$ Our survey sample was randomly selected and national in scope, surveying physicians in 28 states and the District of Columbia. In addition, both pediatricians and family physicians were included. Despite the strengths of the study, there were at least 3 limitations. First, we did not verify through chart review whether self-reported practice reflected actual practice; thus, the true level of care was not independently verified. Second, our study sample was small $(\mathrm{n}=358)$. Last, although the response rate was nearly $60 \%$, and respondents and nonrespondents were demographically similar, it is possible that they differed in their immunization practices.

This study provides a basic framework for understanding physicians' attitudes and practices with regard to adolescent immunization, as well as identifying barriers encountered at the provider level. Improvement is needed in knowledge about varicella vaccine use, use of reminder/recall systems, and overcoming barriers to immunizing older adolescents, particularly among family physicians. 
Education and intervention efforts should be tailored to address specific barriers faced by practitioners and to define the most effective strategies to overcoming these barriers. Further research is warranted to identify practical ways to enhance immunization of all adolescents.

We thank Laura Schild at Emory Center on Health Outcomes and Quality for conducting the randomization process at the initiation of this study and for providing analytical assistance throughout the study.

\section{References}

1. Yusuf H, Averhoff F, Smith N, Brink E. Adolescent immunization: rationale, recommendations, and implementation strategies. Pediatr Ann 1998;27:43644.

2. Averhoff FM, Williams WW, Hadler SC. Immunization of adolescents. Am Fam Physician 1997;55: $159-67$.

3. Immunization of adolescents. Recommendations of the Advisory Committee on Immunization Practices, the American Academy of Pediatrics, the American Academy of Family Physicians, and the American Medical Association. MMWR Recomm Rep 1996; 45(RR-13):1-16.

4. Lannon C, Brack V, Stuart J, et al. What mothers say about why poor children fall behind on immunizations: a summary of focus groups in North Carolina. Arch Pediatr Adolesc Med 1995;149:1070-5.

5. Averhoff FM, Brink E, Pollard B, Resha K, Bryan G, Vaillancourt MV. Adolescent immunization: focus on implementation. J Sch Health 1997;67:304-8.

6. Vernon ME, Bryan G, Hunt P, Allensworth D, Bradley B. Immunization services for adolescents within comprehensive school health programs. J Sch Health 1997;67:252-5.

7. Nichol KL, Mac Donald R, Hauge M. Factors associated with influenza and pneumococcal vaccination behavior among high-risk adults. J Gen Intern Med 1996;11:673-7.
8. Taylor JA, Darden PM, Slora E, Hasemeier CM, Asmussen L, Wasserman R. The influence of provider behavior, parental characteristics, and a public policy initiative on the immunization status of children followed by private pediatricians: a study from pediatric research in office settings. Pediatrics 1997; 99:209-15.

9. Adult immunization: knowledge, attitudes, and practices-DeKalb and Fulton Counties, Georgia, 1988. MMWR Morb Mortal Wkly Rep 1988;37:657-61.

10. Prevention of varicella: recommendations of the Advisory Committee on Immunization Practices (ACIP). Centers for Disease Control and Prevention. MMWR Recomm Rep 1996;45(RR-11):1-36.

11. Licensure of varicella virus vaccine, live. MMWR Morb Mortal Wkly Rep 1995;44:264.

12. Ehresmann KR, Mills WA, Loewenson PR, Moore $\mathrm{K}$. Attitudes and practices regarding varicella vaccination among physicians in Minnesota: implications for public health and provider education. Am J Public Health 2000:90;1917-20.

13. American Academy of Pediatrics, Division of Child Health Research. Periodic Survey of Fellows \#35Adults immunity still a concern. Most fellows offer varicella vaccine; rates vary by location, payment system [monograph on the Internet]. Elk Grove Village (IL): American Academy of Pediatrics; 1998 [cited 2004 Dec 13]. Available from: http://www.aap. org/research/periodicsurvey/ps36b.htm

14. Schaffer SJ, Bruno S. Varicella immunization practices and the factors that influence them. Arch Pediatr Adolesc Med 1999;153:357-62.

15. Newman RD, Taylor JA. Reactions of pediatricians to the recommendation for universal varicella vaccination. Arch Pediatr Adolesc Med 1998;152:792-6.

16. MacFarlane LL. Sanders ML, Carek PJ. Concerns regarding universal varicella immunization. Time will tell. Arch Fam Med 1997;6:537-41.

17. Schaffer SJ, Humiston SG, Shone LP, Averhoff F, Szilagyi PG. Adolescent immunization practices: a national survey of US physicians. Arch Pediatr Adolesc Med 2001;155:566-71. 